\title{
Independent dual-responsive Janus chromic fibers
}

\author{
Yanfang Wei ${ }^{1}$, Wei Zhang ${ }^{1}$, Chengyi Hou ${ }^{1 *}$, Qinghong Zhang ${ }^{2}$, Yaogang $\mathrm{Li}^{2}$ and Hongzhi Wang ${ }^{1 *}$
}

\begin{abstract}
Daily exposure under solar ultraviolet (UV) and infrared (IR) is prone to cause skin cancer and photoaging. Real-time monitoring of the environmental UV index and IR radiation temperature during outdoor activities can enhance awareness and strengthen personal protection. It is a challenge to design flexible, wearable devices (with measurement capabilities) that can be integrated with apparels. Here, microfluidic spinning technology (MST) was used for the continuous and large-scale fabrication of eco-friendly coresheath Janus fibers with a well-defined axially symmetric Janus core. One side of the core was sensitive to UV light and the opposite was sensitive to IR radiation. Textiles woven with Janus fibers showed excellent independent reversible color responses to dual-wavelength stimulation. Such textiles switched among four colors under UV and IR irradiation, both individually and in combination. The color gradient of the textiles changed significantly with increasing UV intensity (UV index). After 60 cycles of UV/IR stimulation and 50 washes, the change rate of the comprehensive chromatic aberration $\left(\Delta E_{a b}^{*}\right)$ of the textiles under different conditions was only $0.42 \%-4.71 \%$. This was attributed to the unique structure of the fibers. The three-line striped textiles demonstrated the potential of the fibers to be used as wearable energy-free realtime visual monitors of the UV index and IR radiation temperature.
\end{abstract}

Keywords: microfluidic spinning, UV index, IR radiation temperature, dual-wavelength response, core-sheath, Janus fibers

\section{INTRODUCTION}

The sun transmits energy to the earth in the form of electromagnetic waves (EMW). Solar radiation (SR) at the ground comprises over $6 \%$ of ultraviolet (UV) radiation $(<400 \mathrm{~nm}), 38 \%-39 \%$ of visible radiation $(400-760 \mathrm{~nm})$, and approximately $54.3 \%$ of infrared (IR) radiation $(760-3000 \mathrm{~nm})[1,2]$. Although UV rays are good for human body, intense UV rays can burn skin and induce skin cancer [3-6]. IR radiation, one of the strongest sources of electromagnetic radiation in our environment, causes the significant thermal radiation effect subsequently with the rapid heat-up of skin, as well as increases the epidermal water evaporation, the skin damage and skin aging, which further promotes the development of skin cancer [7-10]. The intense destruction of the ozone layer and the global greenhouse effect have caused a gradual increase in the energy of the UV radiation from the sun to the earth's surface and the IR radiation in the earth-atmosphere system. Since this seriously affects human health and normal outdoor life [11-13], there is interest in the real-time visual monitoring of the outdoor UV index and the IR radiation temperature. Therefore, there is a requirement for the development of flexible, wearable sensing materials which are sensitive to UV and IR radiation.

Portable technology to monitor the personal UV index and IR radiation temperature can be achieved using comfortable, soft, flexible, biocompatible, breathable, and wearable sensors, which require fibers or textiles as carriers [14]. Portable single- or multi-function devices, which are currently available, are expensive and inevitably consume high energy and additional power [14-16]. Some chemical dyes, which could change color under UV and IR irradiation, were also used to achieve naked-eye monitoring. Commercial photochromic and thermochromic dyes have completely mature and independent chromic mechanisms (see Note S1 in the Supplementary information). Some researchers have integrated photochromic or thermochromic dyes in wearable monitoring devices such as skin patches [15,17], core-sheath fibers [18-21] and textiles [22] by wet spinning [21,23], electrospinning [24], melt spinning [25], screen printing [26], grafting [27,28], and coating [29]. Since these chromic

\footnotetext{
${ }^{1}$ State Key Laboratory for Modification of Chemical Fibers and Polymer Materials, College of Materials Science and Engineering, Donghua University, Shanghai 201600, China

${ }^{2}$ Engineering Research Center of Advanced Glasses Manufacturing Technology, College of Materials Science and Engineering, Donghua University, Shanghai 201600, China

*Corresponding authors (emails: hcy@dhu.edu.cn (Hou C); wanghz@dhu.edu.cn (Wang H))
} 
dyes can be easily rendered ineffective, they are usually encapsulated in polymers such as core layers of coresheath fibers [18-21]. This enhances their durability and practical performance by hindering unnecessary reactions [19]. To realize the innovative monitoring of the UV index and IR radiation temperature simultaneously and independently in a single fiber, the chromic dyes and substrates in these devices need to be carefully structured. It is a great challenge to solve the technological barrier for achieving such designs because they will involve complex fiber structures.

Janus materials have two different compositions and functions which are compartmentalised into two halves of the same object. Janus fibers possess dual functions due to their anisotropic structures [30,31], which arise due to the clear region division on both sides. Therefore, they have attracted the attention of academia and industry [32]. To obtain dual-responsive chromic fibers, the two sides of the Janus interface must be sensitive to UV and IR radiation, respectively. However, there are limited methods to prepare such materials. Electrospinning is the most traditional method for making Janus fibers [33-35]; however, only fiber products like nonwoven mats can be obtained. It is difficult to obtain nano-fiber filaments or short fibers separated from each other. In comparison, microfluidic technology can potentially be used to prepare Janus fibers because it can accurately manipulate small amounts of fluid in an integrated flow channel $[36,37]$. This allows for precise adjustment of the components and the micro-structure of a single fiber [38-40]. The phenomenon of laminar flow in the microchannels ensures easy control of the diversiform cross-sectional shapes of the fibers (circular, beaded, knotted, anisotropic, core sheath, and Janus) $[36,37,41]$. The microfluidic spinning fibers are long, thin, and soft products [38], which are suitable for inexpensively weaving soft and wearable intelligent textiles.

We designed an inexpensive and reusable four-channel microfluidic device. By adjusting the various parameters of the four fluids and the corresponding microchannels, we successfully prepared novel dual- and wide-wavelength-responsive chromic core-sheath fibers with an axially symmetric Janus core, thus facilitating the independent responsiveness. The sheath layer consisted of polylactic acid (PLA). The core layer was an axially symmetric Janus cylinder in which one half comprised polyvinyl alcohol (PVA) mixed with photochromic dyes, and the other half comprised a PVA polymer mixed with thermochromic dyes. Both PLA and PVA promote resource recycling and are ideal eco-friendly polymer ma- terials. The textile woven with such Janus fibers showed excellent reversible color changes with independent dualwavelength responses. The textile could be switched among four colors under UV/IR irradiation. The fibers and textiles also possessed excellent mechanical properties. The comprehensive chromatic aberration $\left(\Delta E_{\mathrm{ab}}^{*}\right)$ before and after textile color changes remained stable, both after 60 cycles of radiation stimulation and 50 washes. The above advantages will endow the fiber with practical application. We designed a three-line striped textile that could be sewn on clothing to achieve energyfree, real-time visual monitoring of the UV index and IR radiation temperatures. Our study is among the few to harness microfluidic spinning technology (MST) to produce core-sheath Janus fibers which have dual independent responses to UV and IR radiation, both individually and in combination.

\section{EXPERIMENTAL SECTION}

\section{Materials}

PLA resin (number-average molecular weight $\left(M_{\mathrm{n}}\right)=1.6$ $\times 10^{5} \mathrm{~g} \mathrm{~mol}^{-1}$, polydispersity index $(\mathrm{PDI})=1.2$, density $=$ $1.2 \mathrm{~g} \mathrm{~cm}^{-3}$, melting point $=176.4^{\circ} \mathrm{C}$ ) was supplied by Nantong Jiuding Biological Engineering Co., Ltd. (China). PVA (weight-average molecular weight $\left(M_{\mathrm{W}}\right)$ $\sim 145,000,>99 \%$ hydrolysed) was purchased from Shanghai Yishi Chemical Technology Co., Ltd. (China). Photochromic and thermochromic dyes were purchased from Zhongshan Longou Dragon Technology Co., Ltd. (China). Analytically pure dichloromethane (DCM) was purchased from Shanghai Lingfeng Chemical Reagent Co., Ltd. (China). Chemically pure polyethylene glycol 400 (PEG 400) was purchased from Sinopharm Chemical Reagent Co., Ltd. (China). Aqueous solutions were prepared with ultrapure water (UPW; resistivity = 18.2 $\mathrm{M} \Omega \mathrm{cm}$ ) obtained from a Milli-Q water purification system (Millipore Corporation, USA), unless stated otherwise. Polytetrafluoroethylene (PTFE) tubes (Shanghai Minji Rubber \& Plastic Products Co., Ltd., China) with inner diameters of $0.6,1$ and $2.6 \mathrm{~mm}$ were used as microchannels.

\section{Design of microchannels}

We used transparent PTFE capillaries and hard steel needles with different diameters to build simple and reusable four-channel microfluidic devices. There was a sequential increase in the microchannel diameters of the inner water phase $(0.6 \mathrm{~mm})$, the middle oil phase $(1 \mathrm{~mm})$, and the outer water phase $(2.6 \mathrm{~mm})$. A detailed descrip- 
tion of the device fabrication is provided in Fig. S1.

\section{Preparation of independent dual-responsive Janus chromic fibers}

Independent dual-responsive Janus chromic fibers with core-sheath structures were prepared by a one-step process using designed microchannels. In this experiment, aqueous solutions of PVA (13 wt\%) containing photochromic dyes (6wt\%) and PVA (13 wt\%) containing thermochromic dyes (6 $\mathrm{wt} \%)$ were used as the inner water phases. A PLA solution (20 wt\%) dissolved in DCM was used as the middle oil phase. PEG 400 was used as the outer water phase. These four solutions were individually poured into four syringes and pumped into designed PTFE microchannels at different rates $\left(v_{\mathrm{PVA}} \sim 1-3 \mathrm{~mL} \mathrm{~h}^{-1}\right.$, $v_{\mathrm{PLA}} \sim 3-6 \mathrm{~mL} \mathrm{~h}^{-1}$, and $v_{\mathrm{PEG}} \sim 80-160 \mathrm{~mL} \mathrm{~h}^{-1}$ ) using an infusion pump (Model 22, Harvard Apparatus, USA); subsequently, the cured functional core-sheath fibers with axially symmetric Janus cores were obtained from the outer microchannel. The white photochromic dyes and the black, green, and blue thermochromic dyes were used as model dyes.

\section{Collection of independent dual-responsive Janus chromic fibers}

We used a simple rotary collector to collect the fibers. Initially, the fibers were evenly drawn by the rotary to enhance their strength; subsequently, the collected fibers were cleaned with deionized water at room temperature for three times, and dried at $50^{\circ} \mathrm{C}$ for $5 \mathrm{~h}$. Finally, they were interwoven as warp and weft threads to obtain a functionally independent dual-responsive chromic textile with the two sides sensitive to IR and UV radiation, respectively.

\section{Characterization and measurements}

The digital photographs of the independent dualresponsive Janus chromic fibers were captured with a digital camera (Nikon D7000, Japan). Optical images were captured using a super deep scene microscope (DVM6/DVM6, Caikon, China) and a laser scanning confocal microscope (TCS SP5 II, Leica, China). The cross section and surface of the fibers were observed by scanning electron microscopy (SEM; Hitachi S-3000, Hitachi, Japan). To assess the UV-sensitive performance of the chromic textiles, the samples were irradiated with a solar simulator (Oriel newport 69911, Oriel, USA) to induce a color change. To assess the IR-sensitive performance of the chromic textiles, the samples were placed on a constant temperature platform (Jingzhi, D20, China) and thermal radiated to induce a color change. The spectral reflectance and $\Delta E_{\mathrm{ab}}^{*}$ were obtained using a desktop color spectrophotometer (CS-820N, CHNspec, China). The average surface temperature of the fabrics woven with the Janus chromic fibers was measured using an FLIR A300 (Oregon, USA), and the Instron 5969 materials testing instrument (Instron, MA, USA) was used for tensile testing of the fibers. In the washing resistance test, we placed the textiles $(5 \mathrm{~cm} \times 4 \mathrm{~cm})$ and the magnetic stirrer into the beakers which contained $200 \mathrm{~mL}$ of household detergent solution ( $4 \mathrm{wt} \%)$. The rotation speed was set to $500 \mathrm{rmin}^{-1}$ and the textiles were washed for $30 \mathrm{~min}$ each time. To test their cyclic color change performances, the textiles were rinsed with pure water after washing to remove any residual detergent. The washing and testing were repeated 50 times.

\section{RESULTS AND DISCUSSION}

In this study, we utilized MST to fabricate new independent dual-responsive Janus chromic fibers with controllable diameters. The fabrication process of the Janus fibers is shown in Fig. 1a-c and Note S2. Two inner water-phase spinning solutions exhibited laminar flow and flowed side by side into a microchannel before entering the middle PLA spinning solution (Fig. 1b). The hydrodynamic focusing effect resulted in the formation of a coaxial flow stream when the two miscible liquids merged and were sheathed by the middle PLA stream $[38,42,43]$. Diffusion-based solvent extraction occurred at the two-phase interface [21,36-40] when the coaxial flow jet entered the collection capillary and encountered the fast-flowing outer PEG 400 phase (coagulation bath) (Fig. 1c, d). Thus, cured functional core-sheath fibers with axially symmetric Janus cores were obtained [36,39].

The key to the well-defined axially symmetric Janus core is the laminar flow maintained by the streamlined microchannel (Fig. 1b, c). The Reynolds number $(R e)$ denotes the ratio of the inertial to viscous forces, which characterizes the tendency of fluids to exhibit either turbulent or non-turbulent (i.e., laminar) flow [42]:

$R e=\frac{\rho v d}{\mu}$,

where $v, \rho$, and $\mu$ are the flow rate in $\mathrm{m} \mathrm{s}^{-1}$, density in $\mathrm{kg} \mathrm{m}^{-3}$, and viscosity of the fluid in $\mathrm{kg} \mathrm{m}^{-1} \mathrm{~s}^{-1}$, respectively; $d$ is the diameter of the capillary in m. $\rho$ and $\mu$ of the fluid were determined as the fluid flowing through the circular channel. When the fluid flow rate was stable, we used channels with smaller $d$. This lowered the Re, which resulted in laminar flow of liquids in the capillaries. Thus, two or more streams converged into a narrow channel by 


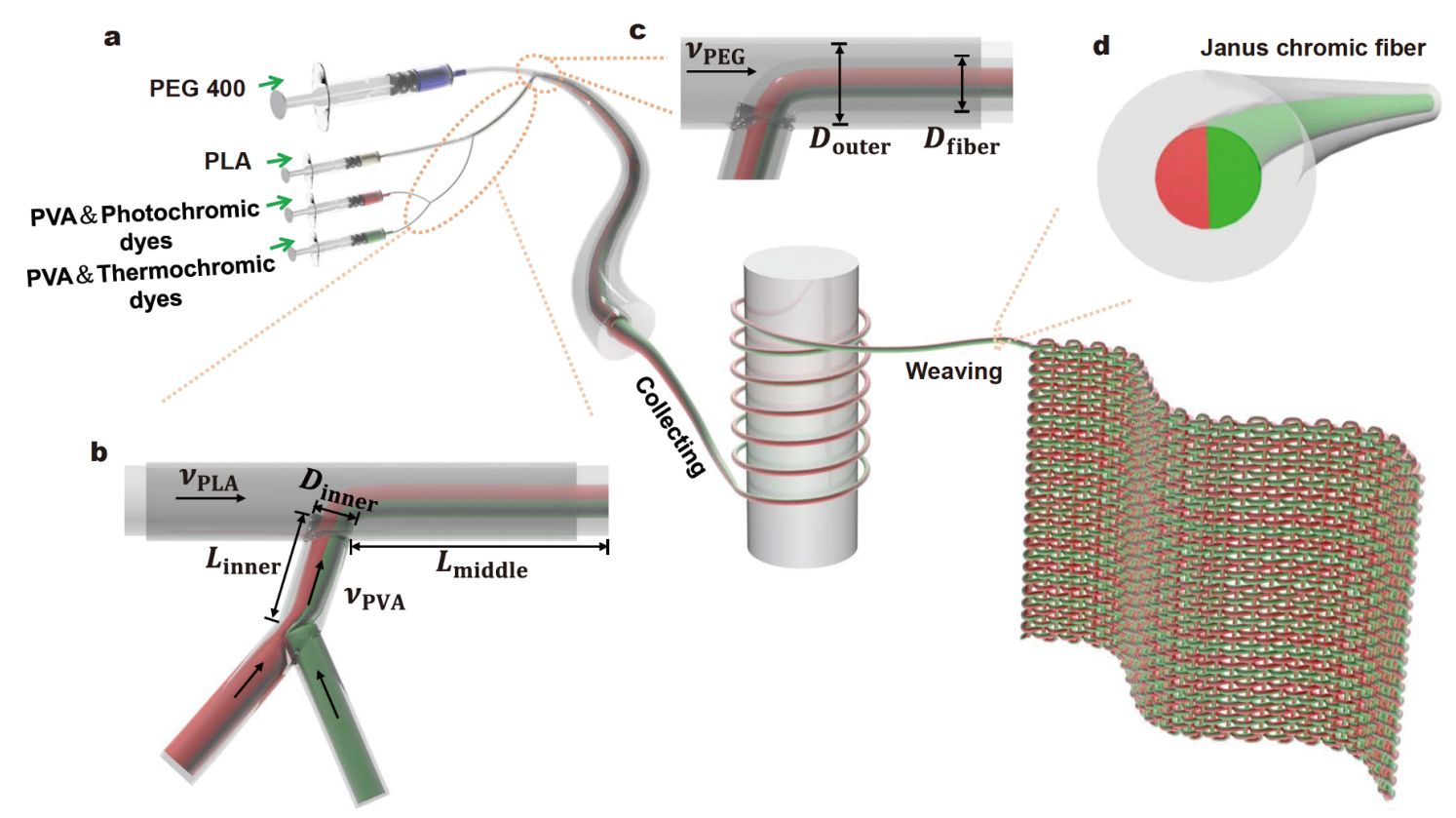

Figure 1 Schematic of MST for preparing independent dual-responsive Janus chromic fibers. (a) Preparation of the fibers and textile. (b, c) Schematic of the generation of the Janus (b) and core-sheath (c) structures by microfluidic devices. (d) Structural scheme of the fibers.

laminar, parallel flow without turbulent mixing [40].

Miscible fluids can only mix by diffusion which forms a dynamic interface (Fig. S2a). Therefore, the influence of transverse diffusion must also be considered along with the maintenance of laminar flow. In the steady state near the top and bottom walls of the interface of the two miscible fluids, the extent of the transverse diffusive mixing $\left(T_{\text {dif }}\right)$ across the fluid-fluid interface is directly proportional to the cube root of the total axial distance along $\left(L_{\text {inner }}+L_{\text {middle }}\right)$ the microchannel and inversely proportional to the cube root of the maximum fluid flow rate $\left(v_{\max }\right)[43]$.

$T_{\text {dif }} \propto\left(\frac{L_{\text {inner }}+L_{\text {middle }}}{v_{\max }}\right)^{1 / 3}$.

In this experiment, the extent of the $T_{\text {dif }}$ was determined by the cumulative degree of diffusion mixing of the inner phases (Fig. 1b, c). $T_{\text {dif }}$ can be measured as a function of the total length of $L_{\text {inner }}$ and $L_{\text {middle }}$ when the fluid flow rate is constant $\left(v_{\mathrm{PVA}} \sim 1-3 \mathrm{~mL} \mathrm{~h}^{-1}\right.$ and $v_{\mathrm{PLA}}$ $\sim 3-6 \mathrm{~mL} \mathrm{~h}^{-1}$ ). Under the condition that the length of the middle phase microchannel was constant $\left(L_{\text {middle }} \sim 2 \mathrm{~cm}\right.$ ), the diffusion of the two miscible fluids increased with an increase in the length of the inner phase microchannel (Fig. S2b, c). Therefore, the diffusion of the two miscible fluids can be decreased before the coaxial flow stream met the outer extraction flow PEG 400 by shortening the
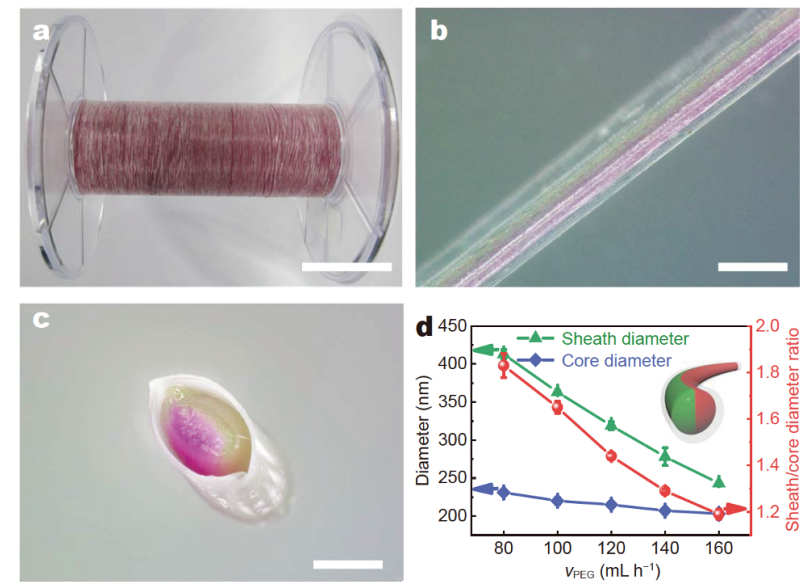

Figure 2 Morphology and structure of the independent dual-responsive Janus chromic fibers. (a) Digital photograph of the fibers. (b, c) Optical images of the outer surface (b) and cross-section (c) of the fibers under the super deep scene microscope. (d) Sheath/core diameter and its diameter ratio curves at different flow rates of PEG 400. Scale bar: (a) $6 \mathrm{~cm}$; (b) $300 \mu \mathrm{m}$; (c) $300 \mu \mathrm{m}$. The error bars correspond to the standard deviation caused by the statistical uncertainty in the measurement.

length of the microchannels of the inner water phase and the middle oil phase (such as: $L_{\text {inner }} \sim 1 \mathrm{~cm}$ and $L_{\text {middle }}$ $\sim 2 \mathrm{~cm}$ in this experiment). This allowed us to precisely control the laminar flow and reduce diffusion to form core-sheath Janus fibers with a well-defined axially symmetric Janus core (Fig. 1d and Fig. S3). 
Fig. 2a-c show the optical images of the Janus chromic fibers fabricated by MST. The digital images demonstrated that the use of MST ensured that the core-sheath Janus fibers could be continuously spun to produce a well-defined Janus structure (Fig. S4a-c). The axially symmetric Janus core comprised two approximately symmetrical quasi-semi-circular fibers assembled shoulder-by-shoulder. One side of the Janus fiber was a thermochromic PVA coaxial fiber comprising PVA mixed with thermochromic dyes. The other side was a photochromic PVA fiber comprising PVA mixed with photochromic dyes. The cross-section and surface structures of the fibers were further observed by SEM (Fig. S4d-h). The fibers possessed uniform sheath thickness and a well-defined core-sheath structure with a PVA chromic core. This proved that the PVA chromic layer was successfully encapsulated in the fiber during its fabrication (Fig. S4d, e, i). The nano-chromic dye particles in the core layer were evenly dispersed (Fig. S4f). In addition, the fiber diameter was uniform, and the sheath surface was extremely smooth without visible impurities (Fig. S4g, h). The PLA sheath of the fiber was almost transparent due to its amorphous structure. Therefore, the green and red parts of the axially symmetric Janus core were clearly visible under the microscope (Fig. 2b, c). It should be mentioned that the PVA sheath layer hardly affected the color change of the PVA core layer (Fig. S5). Therefore, it is possible to use streamlined coaxial microfluidic chips to efficiently produce quasi-cylindrical core-sheath microfibers by MST.

The fiber diameter directly affects the radius of curvature under maximum bending of fiber during production and processing [44]. Therefore, it is critical to achieve precise control of the fiber diameter, which is often affected by the microchannel diameter, fluid flow rate, curing methods, and time [38]. In theory, the diameter of a fiber $\left(D_{\text {fiber }}\right)$ can be denoted as a function of the diameter of the outer water phase microchannel $\left(D_{\text {outer }}\right)$, the volume flow rates of the PEG 400 extraction stream, and the coaxial flow stream $[36,45]$.

$D_{\text {fiber }}=D_{\text {outer }}\left[1-\left(\frac{1}{Q_{\text {coaxial }} / Q_{\mathrm{PEG}}+1}\right)^{1 / 2}\right]^{1 / 2}$,

where $Q_{\text {coaxial }}$ and $Q_{\mathrm{PEG}}$ are the volume flow rates of the coaxial flow stream and PEG 400 extraction stream, respectively. Here, we explored the effect of different flow rates of PEG $400\left(v_{\mathrm{PEG}}\right)$ on the fiber diameter and the sheath/core diameter ratio. It was found that when the $v_{\mathrm{PVA}}$ and $v_{\mathrm{PLA}}$ were fixed, the increase in $v_{\mathrm{PEG}}$ contributed to the decreasing sheath diameter, core diameter, and sheath/core diameter ratio (Fig. 2d). However, the sheath diameter decreased faster than the core diameter because the shear stress on the surface of the middle phase fluid PVA increased with an increase in $v_{\mathrm{PEG}}$, which resulted in a smaller fiber diameter $[21,36,40]$. Therefore, the fiber sheath was thinner than the core. It is known that the diameter and the sheath/core diameter ratio of the fibers can be precisely regulated by adjusting the flow rate in the microchannel. Therefore, by using MST and adjusting various parameters, we precisely regulated the morphology of the fibers to ensure structural stability and consistency in the morphology of the prepared core-sheath Janus chromic fibers.

To elucidate the color change of the Janus chromic fibers, we tested and recorded the reversible color change of the cross-sectional structure of the fibers at the microscopic level. Both sides of the Janus fiber always exhibited two distinct colors under different radiation conditions (Fig. 3). When the test environment temperature was maintained at approximately $18^{\circ} \mathrm{C}$, the UVresponsive and the IR-responsive halves of the Janus fiber core layer were white and grey, respectively, in the absence of additional radiation. UV radiation $(\lambda=$ $280-400 \mathrm{~nm}$ ) turned the UV-responsive half of the fiber red. The thermal effect raised the temperature of the fibers to $30^{\circ} \mathrm{C}$, which turned the IR-responsive half of the fiber green.

The chromic Janus fibers were woven into textiles to demonstrate their color-changing abilities. Fig. 4 shows the color of the chromic textile under different conditions. It was grey $\left(18^{\circ} \mathrm{C}\right)$, wine red $\left(18^{\circ} \mathrm{C}\right.$ and UV), green $\left(30^{\circ} \mathrm{C}\right)$, and bright red $\left(30^{\circ} \mathrm{C}\right.$ and $\left.\mathrm{UV}\right)$ on a macro scale, and showed good reversibility (Fig. 4a and Video S1). The
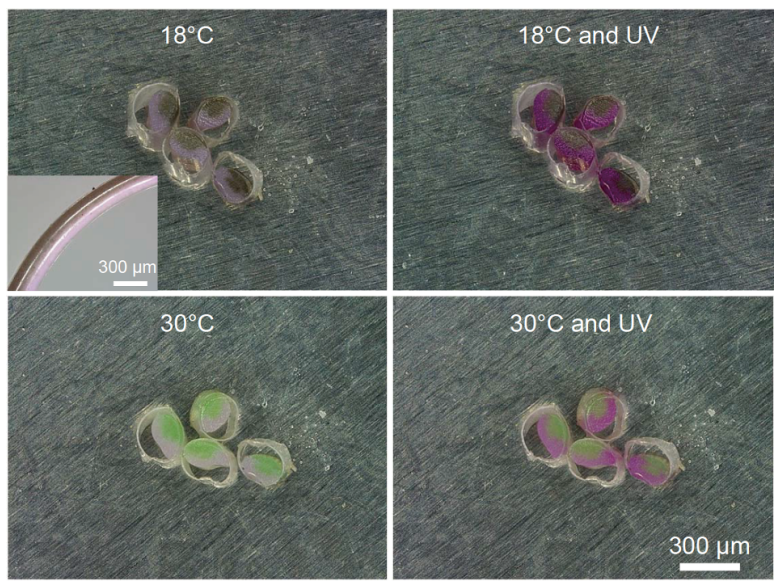

Figure 3 The cross-sectional morphologies of the fibers under different conditions under the super deep scene microscope. 

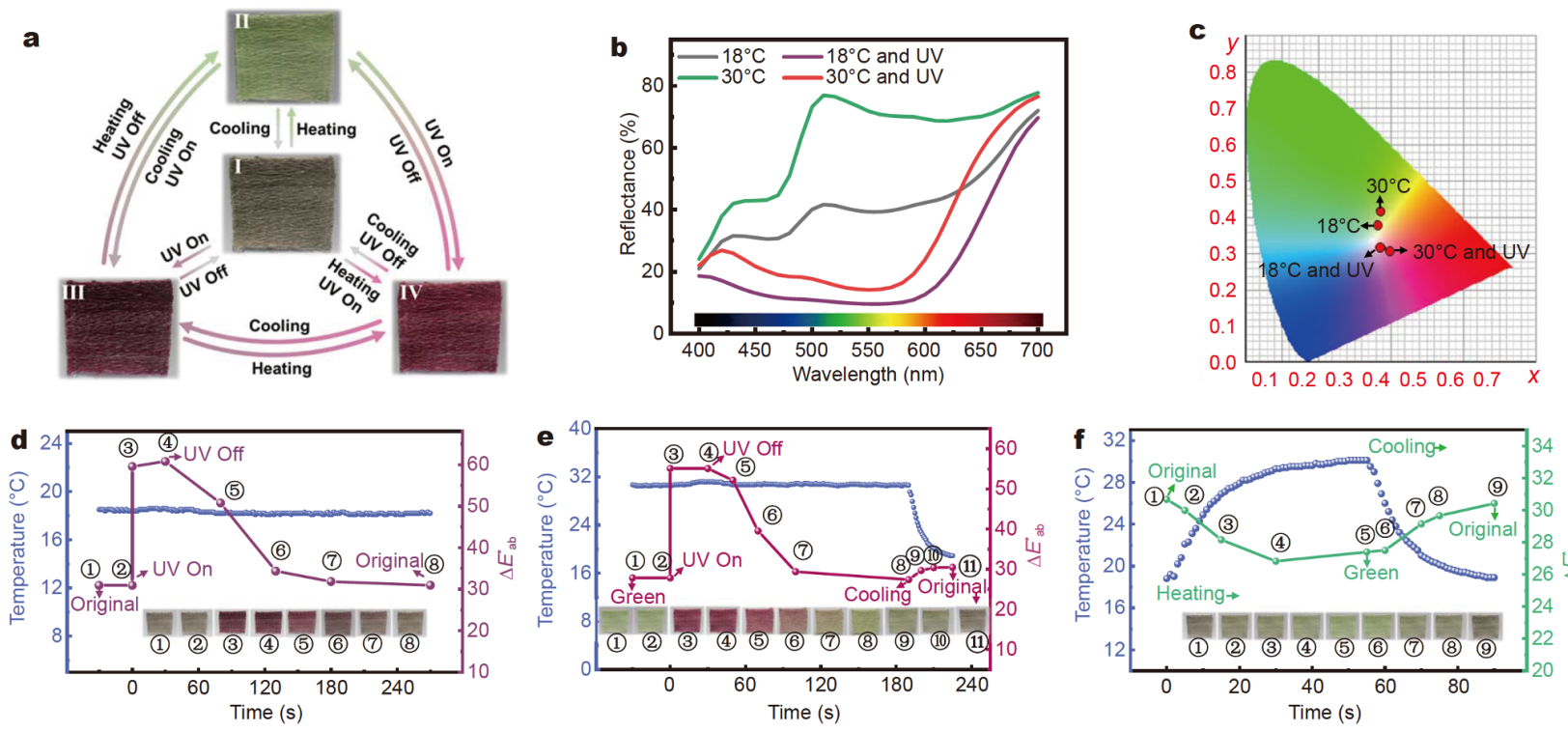

Figure 4 Independent chromic behavior of chromic textiles. (a) Digital photographs of the reversible color change of the textile under different conditions (the size of textile is $5 \mathrm{~cm} \times 4 \mathrm{~cm}$ ). (b) The reflectance curves of the textile under different conditions. (c) The color distribution of the textile under different conditions in the $(x, y)$ chromaticity diagram (standard CIE 1931). (d) Curves of $\Delta E_{\mathrm{ab}}^{*}$ and temperature when the textile underwent photochromism under UV irradiation at $18^{\circ} \mathrm{C}$. (e) Curves of $\Delta E_{\mathrm{ab}}^{*}$ and temperature when the textile underwent photo-thermochromism under UV irradiation at $30^{\circ} \mathrm{C}$. (f) Curves of $\Delta E_{\mathrm{ab}}^{*}$ and temperature when the textile underwent thermochromism on heating and cooling.

visible light reflectance of the textile (Fig. $4 \mathrm{~b}$ ) shows that the difference between its reflectance curves lay in the position of the reflectance peak and the reflectance intensity under different conditions. Though approximately every reflectance curve has two spectral peaks, the textile color was determined by the first peaks since the peaks at high wavelengths were imperceptible to humans [15]. The four reflectance curves represented the colors of the chromic textile under different conditions, i.e., grey $\left(18^{\circ} \mathrm{C}\right)$, wine red $\left(18^{\circ} \mathrm{C}\right.$ and UV), green $\left(30^{\circ} \mathrm{C}\right)$, and bright red $\left(30^{\circ} \mathrm{C}\right.$ and $\left.\mathrm{UV}\right)$. And the diameter of the fibers did not affect their chromic performance (Fig. S6). The colors of textile under different conditions were recorded in the CIE 1931 chromaticity diagram (Fig. 4c). The color coordinates of the textile at $18^{\circ} \mathrm{C}$ in the chromaticity diagram were determined to be $(0.3416,0.3598)$, which indicated that the textile was grey and slightly green. At $(0.3531,0.2926)$ under UV irradiation, the textile turned green $(0.344,0.3885)$ when IR radiation raised the temperature to $30^{\circ} \mathrm{C}$. When the textile was irradiated with UV at $30^{\circ} \mathrm{C}$, it became bright red $(0.3583,0.2901)$. A quantitative evaluation of the reversible color changing properties of the chromic textile showed that the color relative to the standard sample (in the experiment, the whiteboard was used as a standard sample) deepened with the increase in $\Delta E_{\mathrm{ab}}^{*}\left(\Delta E_{\mathrm{ab}}^{*}\right.$ stands for comprehensive chromatic aberration relative to the color of the standard sample) (Fig. 4d-f). In this study, we defined the response time of the Janus fiber as the time required for $90 \%$ of the color change between the bleached and colored states to occur $[46,47]$. When the textile was irradiated with a solar simulator at $18^{\circ} \mathrm{C}, \Delta E_{\mathrm{ab}}^{*}$ of the textile increased instantly (the colored time was about $10 \mathrm{ps}$ ) [48], which indicated the change from grey to wine red (Fig. 4d). When the solar simulator was turned off after $10 \mathrm{~s}, \Delta E_{\mathrm{ab}}^{*}$ of the textile gradually decreased, which indicated that it had started bleaching. At 18 and $30^{\circ} \mathrm{C}$, the bleached times of the textile were calculated to be approximately 243 and $200 \mathrm{~s}$, respectively (Fig. $4 \mathrm{~d}$, e). Under IR thermal radiation, $\Delta E_{\mathrm{ab}}^{*}$ of the textile decreased gradually, which indicated the gradual change from grey to green (Fig. 4f). The colored and bleached times of the textile were calculated to be approximately 49 and $31 \mathrm{~s}$. UV radiation hardly changed the surface temperature of the textile (Fig. 4d, e). Therefore, UV and IR radiation independently caused thermochromism of the textile without interfering with each other.

We assessed the capability of the Janus chromic fibers in monitoring the UV intensity (UV index) by exploring the effect of the UV intensity on the chromic behaviour of the textiles. This was based on the global UV index and the UV intensity classification standard (Fig. 5a) [2]. The UV intensity was controlled by adjusting the distance between the light source and the textile and calibrated 


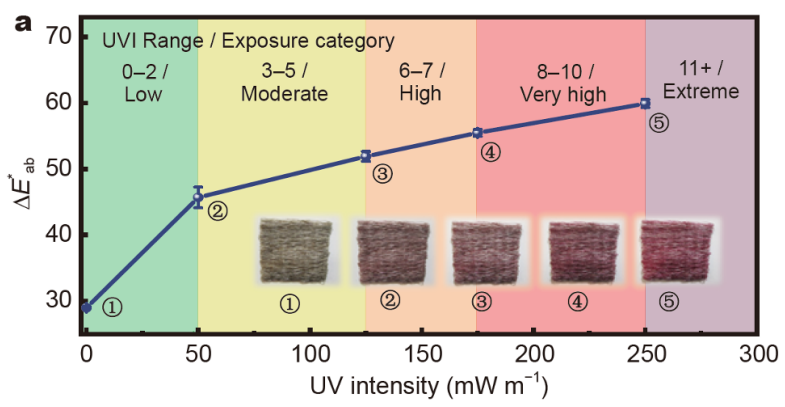

b

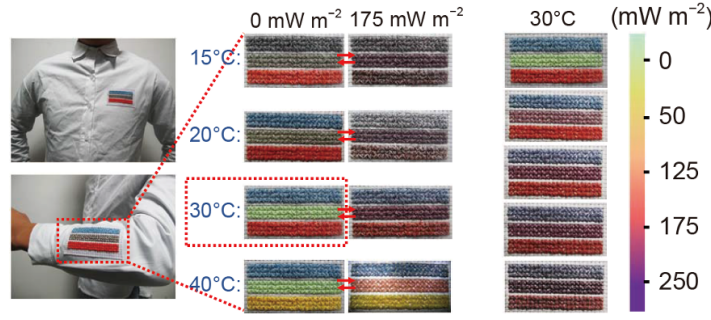

Figure 5 Real-time monitoring capability of chromic textiles. (a) Color change of the chromic textile under different UV intensities at a stable temperature of $18^{\circ} \mathrm{C}$ (the size of textile is $5 \mathrm{~cm} \times 4 \mathrm{~cm}$ ). The error bars correspond to the standard deviation caused by the statistical uncertainty in the measurement. (b) Digital photographs of a three-line striped textile displaying the color gradient change for proof-of-concept monitoring of the ambient UV index and the IR radiation temperature (the size of textile is $8 \mathrm{~cm} \times 5 \mathrm{~cm}$ ). The UV intensities from top to bottom are: $0,50,125,175$ and $250 \mathrm{~mW} \mathrm{~m}^{-2}$, respectively.

using a solar UV intensity metre (TM213, Tenmars, China). During the test, the fabric was exposed to UV radiation at $18^{\circ} \mathrm{C}$ for $5 \mathrm{~s}$. We found that $\Delta E_{\mathrm{ab}}^{*}$ of the textile increased significantly with an increase in the UV intensity. Moreover, there was a visible change in the color gradient of the textile. Therefore, the textile served as an effective visual indicator of the UV index (UV intensity).

Based on the structural design of the Janus chromic fibers mentioned above, chromic dyes, which were sensitive to different temperatures, were assembled into the fibers to monitor multiple temperatures. UV-sensitive dyes could monitor the UV intensity (Fig. 5a). We designed a three-line striped textile using Janus chromic fibers to demonstrate its efficacy for the real-time visual monitoring of the UV index and the IR radiation temperature in daily life (Fig. 5b). The textile could be sewed onto clothes to allow the three lines of the pattern to monitor different ambient temperatures and UV index (Fig. 5b and Fig. S7). When the ambient temperature was below $20^{\circ} \mathrm{C}\left(\right.$ e.g., $\left.15^{\circ} \mathrm{C}\right)$, the first line of the pattern was black; it turned blue when the temperature reached approximately $20^{\circ} \mathrm{C}$. The second line of the pattern changed from grey to green when the temperature reached ap- proximately $30^{\circ} \mathrm{C}$; when the ambient temperature rose to approximately $40^{\circ} \mathrm{C}$, the third line of the pattern changed from orange to yellow. At a stable ambient temperature of $30^{\circ} \mathrm{C}$, there was a visibly significant change in the color gradient of the textile when the UV intensity increased. This demonstrated that the color of the three-line striped textile varied with the UV intensity at any specific temperature. Therefore, the pattern was capable of energyfree, real-time visual monitoring of the IR radiation temperature and the UV index.

The mechanical properties and bending behaviors of the fibers directly affect their practical performance. Therefore, we successfully fabricated fibers with different sheath/core diameter ratios by adjusting the $v_{\mathrm{PEG}}$ and subjected these fibers to the tensile strength test (Fig. 6a). The results showed that the Janus chromic fibers with low sheath/core diameter ratios possessed high strength. At a sheath/core diameter ratio of 1.19 , the fiber achieved a high strength of about $27 \mathrm{MPa}$ and elastic modulus of about $500 \mathrm{MPa}$. This was due to the increase in the degree of orientation of the PLA molecular chain of the fiber sheath with the increase in $v_{\mathrm{PEG}}$. The tensile strengths of the PLA sheath fibers, PVA core fibers, and PLA-PVA core-sheath fibers, which were spun at an $v_{\mathrm{PEG}}$ of $160 \mathrm{~mL} \mathrm{~h}^{-1}$, were measured (Fig. 6b). The molecular structure of the PVA core fibers ensured that their tensile strength (approximately $20 \mathrm{MPa}$ ) was lower than that of the PLA sheath fibers ( $44 \mathrm{MPa})$. The strength advantage of the core-sheath fibers was highlighted by the fact that their tensile strength $(\sim 27 \mathrm{MPa})$ was higher than that of the PVA fibers. Besides, the fibers with different colors were tied in a knot and twisted (Fig. S8a). The fibers all exhibit good bending performance, which makes it easy to be woven into various patterns. As shown in Fig. S8b, the textile of the Janus chromic fibers was bent in different degrees, which shows that the textile is very soft and can be applied to flexible wearable devices. Therefore, the excellent practicability and functionality of the Janus chromic fibers and their textile were reaffirmed from these observations.

To evaluate the practical performance of the fibers, factors like their color-changing cycle performance and washing ability are also important. The optical chromic textile was highly sensitive to UV/IR radiation. At $18^{\circ} \mathrm{C}$, the textile was subjected to 60 cycles of stimulation under UV and IR irradiation, both individually and in combination. The textile underwent fast and real-time photochromic, thermochromic, and photo-thermochromic switches without any degradation, thereby demonstrating its excellent response rate and cyclic reversibility (Fig. 6c 

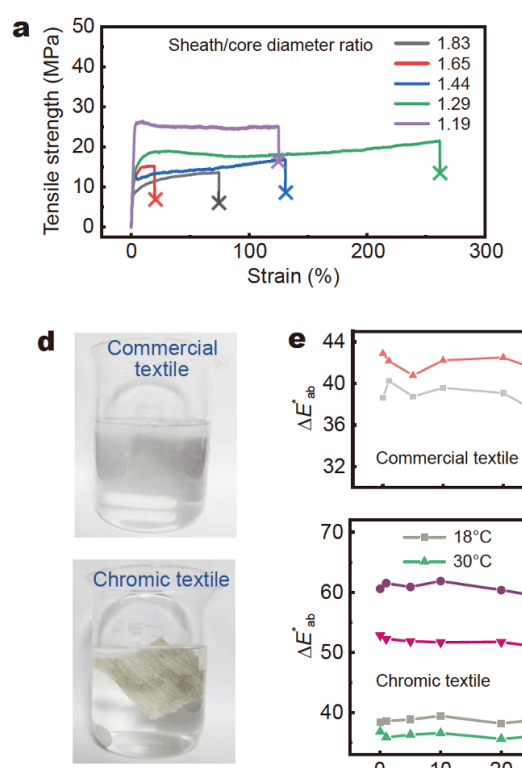

b
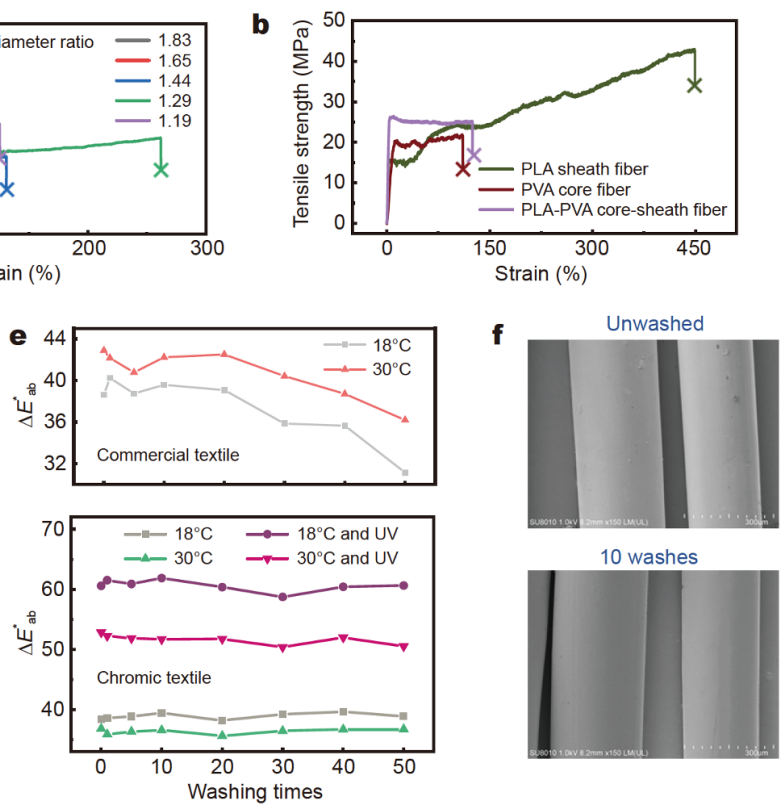

10 washes
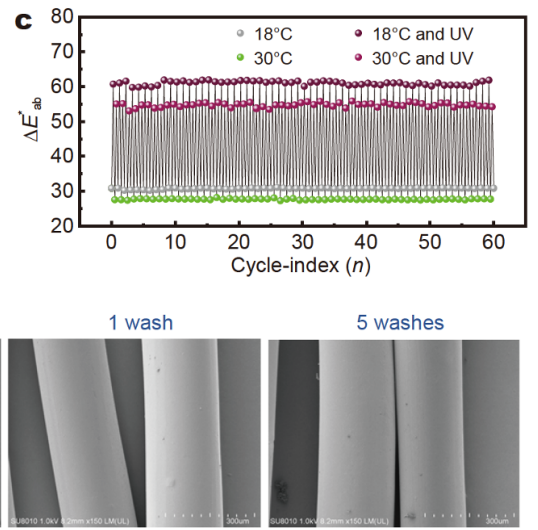

30 washes
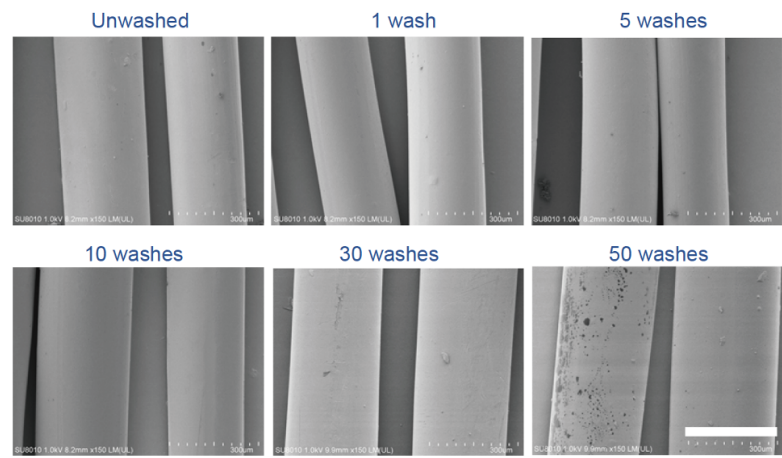

50 washes

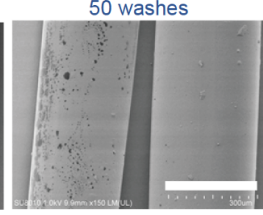

Figure 6 Practicability and durability of chromic textiles. (a) Stress-strain curves of the fibers at different outer flow rates. (b) Stress-strain curves of the PLA sheath fibers, PVA core fibers, and PLA-PVA core-sheath Janus fibers. (c) Relative changes in the colorimetric response after 60 cycles of stimulation at temperatures of 18 and $30^{\circ} \mathrm{C}$ and under UV irradiation at 18 and $30^{\circ} \mathrm{C}$. (d) Digital photographs of the commercial textile (top) and the experimentally fabricated chromic textile (bottom) after 50 washes (both textiles are $5 \mathrm{~cm} \times 4 \mathrm{~cm}$ in size). (e) $\Delta E_{\text {ab }}^{*}$ contrasts of the commercial textile (top) and the experimentally fabricated chromic textile (bottom) after 50 washes. (f) Morphology of the unwashed Janus chromic fibers after being washed $1,5,10,20,30,40$, and 50 times (scale bars: $300 \mu \mathrm{m}$ ).

and Fig. S9). Fig. S10 shows that the chromic performance of the Janus chromic fibers remained unaffected despite being soaked in water. Fig. S11 shows that the water did not affect the UV/IR radiation responsive performance of the fiber. Further, we washed the experimentally fabricated chromic textile and a commercial chromic textile to evaluate the washing ability of the Janus chromic fibers (Fig. 6d-f). The color of the commercial textile without the sheath layer faded significantly after washing (Fig. 6d and Fig. S12) while that of the experimentally fabricated chromic textile did not fade at all. When the commercial textile was subjected to an increasing number of washes, its $\Delta E_{\mathrm{ab}}^{*}$ markedly decreased under different conditions (Fig. 6e); and when we washed the PVA chromic fibers, they swelled, partially broke, and dissolved during the first washing process (Fig. S12). However, $\Delta E_{\mathrm{ab}}^{*}$ and the reflectivity curves of the experimentally fabricated chromic textile remained stable despite being washed 50 times (Fig. 6e and Fig. S13). The SEM images of the unwashed chromic textile after $1,5,10,30$, and 50 washes, respectively. Fig. $6 \mathrm{f}$ shows that the surface of the PVA sheath of the Janus chromic fibers remained smooth without any serious cracks even after 50 washes. This indicated that the
PVA chromic core layer neither overflowed nor got damaged; it was still highly sensitive to UV and IR radiation. This verified the protective effect of the PLA sheath layer on the water-soluble PVA chromic core layer. Therefore, Janus chromic fibers showed excellent washing resistance and stable practical performance.

\section{CONCLUSIONS}

In summary, we successfully designed eco-friendly, independent, dual-responsive Janus chromic fibers and their textiles. The fibers and their textiles showed stable practical performance and outstanding sensitivity to UV/ IR radiation to achieve energy-free, real-time visual monitoring of the IR radiation temperature and UV index. The perfect core-sheath structure of the Janus fibers ensured the feasibility of their textiles. The sheath layer was a highly transparent PLA polymer, while the core layer was an axially symmetric Janus core with a clear interface. The UV-responsive half of the Janus core comprised PVA mixed with photochromic dyes, while the IR-responsive half comprised PVA mixed with thermochromic dyes. Both the sheath and core layers of the fibers were built from degradable materials to promote eco-efficiency and sustainability. The three-line striped 
textiles woven with the Janus chromic fibers exhibited dual-responsive reversible color changes under UV/IR irradiation, which facilitated visual monitoring of the environmental temperature and the UV index. After 60 cycles of stimulation by UV/IR irradiation and 50 washes, the change rate of $\Delta E_{\mathrm{ab}}^{*}$ of the textiles under different conditions was only $0.42 \%-4.71 \%$. The Janus chromic fibers are extremely practicable and can be designed as decorative patterns to be sewn on clothes to effectively protect the human skin from UV/IR radiation. Besides, we have evaluated the cost of all raw materials and chemical reagents used to prepare independent dualresponsive Janus chromic fibers, which is about $\$ 11.65$ per $500 \mathrm{~g}$ (see Table S1 and Note S3).

Received 17 July 2020; accepted 16 November 2020; published online 2 February 2021

1 Zhuang TT, Liu Y, Li Y, et al. Integration of semiconducting sulfides for full-spectrum solar energy absorption and efficient charge separation. Angew Chem Int Ed, 2016, 55: 6396-6400

2 World Health Organization. Global solar UV index: A practical guide: A joint recommendation of WHO, WMO, UNEP and ICNIRP. 2002

3 Dong K, Goyarts E, Rella A, et al. Age associated decrease of MT-1 melatonin receptor in human dermal skin fibroblasts impairs protection against UV-induced DNA damage. Int J Mol Sci, 2020, 21: 326

4 Italia N, Rehfuess EA. Is the global solar UV index an effective instrument for promoting sun protection? A systematic review. Health Education Res, 2012, 27: 200-213

5 Heo SY, Kim J, Gutruf P, et al. Wireless, battery-free, flexible, miniaturized dosimeters monitor exposure to solar radiation and to light for phototherapy. Sci Transl Med, 2018, 10: eaau1643

6 Anandasabapathy N. Sensing sun damage. Sci Transl Med, 2012, 4: $147 \mathrm{ec} 144$

7 Sklar LR, Almutawa F, Lim HW, et al. Effects of ultraviolet radiation, visible light, and infrared radiation on erythema and pigmentation: A review. Photochem Photobiol Sci, 2013, 12: 54-64

8 Hudson L, Rashdan E, Bonn CA, et al. Individual and combined effects of the infrared, visible, and ultraviolet light components of solar radiation on damage biomarkers in human skin cells. FASEB J, 2020, 34: 3874-3883

9 Piil JF, Christiansen L, Morris NB, et al. Direct exposure of the head to solar heat radiation impairs motor-cognitive performance. Sci Rep, 2020, 10: 7812

10 Sánchez-Pérez JF, Vicente-Agullo D, Barberá M, et al. Relationship between ultraviolet index (UVI) and first-, second- and third-degree sunburn using the Probit methodology. Sci Rep, 2019, 9: 733

11 Luber G, McGeehin M. Climate change and extreme heat events. Am J Prev Med, 2008, 35: 429-435

12 McMichael AJ, Lindgren E. Climate change: Present and future risks to health, and necessary responses. J Internal Med, 2011, 270: 401-413

13 Jones B, O'Neill BC, McDaniel L, et al. Future population exposure to US heat extremes. Nat Clim Change, 2015, 5: 652-655

14 Araki H, Kim J, Zhang S, et al. Materials and device designs for an epidermal UV colorimetric dosimeter with near field communication capabilities. Adv Funct Mater, 2017, 27: 1604465

15 Kurz W, Yetisen AK, Kaito MV, et al. UV-sensitive wearable devices for colorimetric monitoring of UV exposure. Adv Opt Mater, 2020, 8: 1901969

16 Polat EO, Mercier G, Nikitskiy I, et al. Flexible graphene photodetectors for wearable fitness monitoring. Sci Adv, 2019, 5: eaaw7846

17 Park B, Kim JU, Kim J, et al. Strain-visualization with ultrasensitive nanoscale crack-based sensor assembled with hierarchical thermochromic membrane. Adv Funct Mater, 2019, 29: 1903360

18 Lu X, Zhang Z, Sun X, et al. Flexible and stretchable chromatic fibers with high sensing reversibility. Chem Sci, 2016, 7: 5113-5117

19 Pan J, Hao B, Xu P, et al. Highly robust and durable core-sheath nanocomposite yarns for electro-thermochromic performance application. Chem Eng J, 2020, 384: 123376

20 Pan J, Hao B, Song W, et al. Highly sensitive and durable wearable strain sensors from a core-sheath nanocomposite yarn. Compos Part B-Eng, 2020, 183: 107683

21 Zhang W, Hou C, Li Y, et al. Microfluidic spinning of editable polychromatic fibers. J Colloid Interface Sci, 2020, 558: 115-122

22 Zhang W, Ji X, Peng BJ, et al. High-performance thermoresponsive dual-output dye system for smart textile application. Adv Funct Mater, 2019, 30: 1906463

23 Pinto TV, Cardoso N, Costa P, et al. Light driven PVDF fibers based on photochromic nanosilica@naphthopyran fabricated by wet spinning. Appl Surf Sci, 2019, 470: 951-958

24 Genovese ME, Caputo G, Nanni G, et al. Light responsive silk nanofibers: An optochemical platform for environmental applications. ACS Appl Mater Interfaces, 2017, 9: 40707-40715

25 Pinto TV, Fernandes DM, Guedes A, et al. Photochromic polypropylene fibers based on UV-responsive silica@phosphomolybdate nanoparticles through melt spinning technology. Chem Eng J, 2018, 350: 856-866

26 Huang G, Liu L, Wang R, et al. Smart color-changing textile with high contrast based on a single-sided conductive fabric. J Mater Chem C, 2016, 4: 7589-7594

27 Zhang W, Ji X, Yin Y, et al. Temperature induced color changing cotton fabricated via grafting epoxy modified thermochromic capsules. Cellulose, 2019, 26: 5745-5756

28 ter Schiphorst J, van den Broek M, de Koning T, et al. Dual light and temperature responsive cotton fabric functionalized with a surface-grafted spiropyran-NIPAAm-hydrogel. J Mater Chem A, 2016, 4: 8676-8681

29 Macharia DK, Ahmed S, Zhu B, et al. UV/NIR-light-triggered rapid and reversible color switching for rewritable smart fabrics. ACS Appl Mater Interfaces, 2019, 11: 13370-13379

30 Wang X, Feng X, Ma G, et al. Amphiphilic Janus particles generated via a combination of diffusion-induced phase separation and magnetically driven dewetting and their synergistic self-assembly. Adv Mater, 2016, 28: 3131-3137

31 Han H, Baik S, Xu B, et al. Bioinspired geometry-switchable Janus nanofibers for eye-readable $\mathrm{H}_{2}$ sensors. Adv Funct Mater, 2017, 27: 1701618

32 Hessberger T, Braun LB, Zentel R. Interfacial self-assembly of amphiphilic dual temperature responsive actuating Janus particles. Adv Funct Mater, 2018, 28: 1800629

33 Yu DG, Wang M, Li X, et al. Multifluid electrospinning for the generation of complex nanostructures. WIREs Nanomed Nanobiotechnol, 2020, 12: e1601 
34 Starr JD, Budi MA, Andrew JC. Processing-property relationships in electrospun Janus-type biphasic ceramic nanofibers. J Am Ceram Soc, 2015, 98: 12-19

35 Yang W, Gong W, Hou C, et al. All-fiber tribo-ferroelectric synergistic electronics with high thermal-moisture stability and comfortability. Nat Commun, 2019, 10: 5541

36 Du X, Li Q, Wu G, et al. Multifunctional micro/nanoscale fibers based on microfluidic spinning technology. Adv Mater, 2019, 31: 1903733

37 Zhang Y, Wang CF, Chen L, et al. Microfluidic-spinning-directed microreactors toward generation of multiple nanocrystals loaded anisotropic fluorescent microfibers. Adv Funct Mater, 2015, 25: 7253-7262

38 Guo J, Yu Y, Wang $\mathrm{H}$, et al. Conductive polymer hydrogel microfibers from multiflow microfluidics. Small, 2019, 15: 1805162

39 Yu Y, Chen G, Guo J, et al. Vitamin metal-organic frameworkladen microfibers from microfluidics for wound healing. Mater Horiz, 2018, 5: 1137-1142

40 Atencia J, Beebe DJ. Controlled microfluidic interfaces. Nature, 2005, 437: 648-655

41 Li Q, Xu Z, Du X, et al. Microfluidic-directed hydrogel fabrics based on interfibrillar self-healing effects. Chem Mater, 2018, 30: 8822-8828

42 Kenis PJA, Ismagilov RF, Takayama S, et al. Fabrication inside microchannels using fluid flow. Acc Chem Res, 2000, 33: 841-847

43 Ismagilov RF, Stroock AD, Kenis PJA, et al. Experimental and theoretical scaling laws for transverse diffusive broadening in twophase laminar flows in microchannels. Appl Phys Lett, 2000, 76: 2376-2378

44 Li K, Zhang Q, Wang H, et al. Lightweight, highly bendable and foldable electrochromic films based on all-solution-processed bilayer nanowire networks. J Mater Chem C, 2016, 4: 5849-5857

45 Zarrin F, Dovichi NJ. Sub-picoliter detection with the sheath flow cuvette. Anal Chem, 1985, 57: 2690-2692

46 Li R, Li K, Wang G, et al. Ion-transport design for high-performance $\mathrm{Na}^{+}$-based electrochromics. ACS Nano, 2018, 12: 37593768

47 Fan $\mathrm{H}, \mathrm{Li} \mathrm{K}$, Liu X, et al. Continuously processed, long electrochromic fibers with multi-environmental stability. ACS Appl Mater Interfaces, 2020, 12: 28451-28460

48 Aramaki S, Atkinson GH. Spirooxazine photochromism: Picosecond time-resolved Raman and absorption spectroscopy. Chem Phys Lett, 1990, 170: 181-186

Acknowledgements This work was supported by the Fundamental Research Funds for the Central Universities (2232019G-02 and 2232019A3-02), Donghua University Distinguished Young Professor Program (LZB2019002), and Shanghai Rising-Star Program (20QA1400300).

Author contributions Wei Y and Zhang W designed and conceived the project, and carried out the preparation, measurement and characterization of independent dual-responsive Janus chromic fibers; Wang H, Hou C, Zhang Q and Li Y guided the project and assisted the result analysis; Wei $\mathrm{Y}$ wrote the manuscript.
Conflict of interest The authors declare that they have no conflict of interest.

Supplementary information Experimental details and supporting data are available in the online version of the paper.

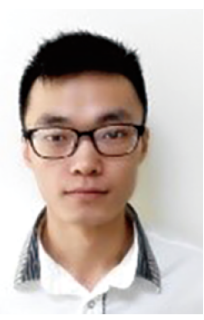

Chengyi Hou received his $\mathrm{PhD}$ degree from Donghua University in 2014, during which he visited to the academician Jens Ulstrup's research group, Department of Chemistry, Technical University of Denmark. He joined the College of Materials Science and Engineering in Donghua University in 2014. In recent years, he mainly engages in the research of organic-inorganic composite systems based on low-dimensional nanomaterials. He designed a series of nanocomposites with environmental response properties, as well as three-dimensional macroscopic materials in the form of fibers, films, and aerogels.

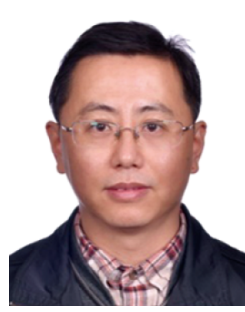

Hongzhi Wang completed his postdoc research at the National Institute of Advanced Industrial Science and Technology, Japan. He joined the College of Materials Science and Engineering in Donghua University as a full professor in 2005 . In recent years, he leads a research group at Donghua University and pursues to construct various flexible multi-functional devices, including flexible optoelectronic devices, artificial muscles and flexible energy sources/systems for smart textiles/clothing.

\section{独立双响应Janus变色纤维}

位艳芳 ${ }^{1}$, 张伟 $^{1}$, 侯成义 ${ }^{1 *}$, 张青红 ${ }^{2}$, 李耀刚 ${ }^{2}$, 王宏志 ${ }^{1^{*}}$

摘要 在户外活动中, 频繁暴露于太阳紫外线和红外辐射下皮肤容 易发生癌变和老化. 对环境中的紫外线强度和红外辐射进行实时 监测有益于增强个人防护. 设计可与服装集成且兼具监测功能的 光响应材料与柔性器件是一项挑战. 本文中, 我们利用微流控纺丝 技术实现了具有轴向对称性皮芯结构Janus变色纤维的连续化制 备. 芯层材料双侧分别对紫外和红外波段光敏感. 用Janus纤维编织 的纺织品对紫外/红外双波段刺激具有独立可逆的变色响应, 使得 纤维能够在四种颜色间切换. 纺织品的颜色能随着光照强度的改 变发生显著的梯度变化. 由于皮层的保护作用, 经过60次紫外/红 外刺激或 50 次洗涤后, 纺织品的综合色差 $\left(\Delta E^{*}{ }_{\mathrm{ab}}\right)$ 变化率仅为 $0.42 \%-4.71 \%$. 我们设计的三线条纹纺织品展示出该纤维可以用作 紫外线指数和红外辐射的可穿戴式无能耗、实时、可视化监测. 